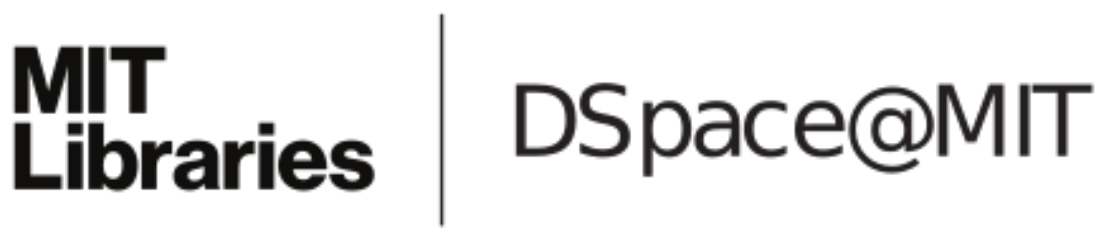

\author{
MIT Open Access Articles
}

\section{Images of Asteroid 21 Lutetia: A Remnant Planetesimal from the Early Solar System}

The MIT Faculty has made this article openly available. Please share how this access benefits you. Your story matters.

Citation: Sierks, H. et al. “Images of Asteroid 21 Lutetia: A Remnant Planetesimal from the Early Solar System." Science 334.6055 (2011): 487-490.

As Published: http://dx.doi.org/10.1126/science.1207325

Publisher: American Association for the Advancement of Science (AAAS)

Persistent URL: http://hdl.handle.net/1721.1/110553

Version: Author's final manuscript: final author's manuscript post peer review, without publisher's formatting or copy editing

Terms of use: Creative Commons Attribution-Noncommercial-Share Alike 


\section{Images of Asteroid 21 Lutetia: A Remnant Planetesimal from the Early Solar System}

H. Sierks ${ }^{1^{*}}$, P. Lamy ${ }^{2}$, C. Barbieri ${ }^{3,32}$, D. Koschny ${ }^{4}$, H. Rickman ${ }^{5,31}$, R. Rodrigo ${ }^{6}$, M. F. A'Hearn ${ }^{7}$, F. Angrilli, ${ }^{8,32}$, M. A. Barucci ${ }^{9}$, J.-L. Bertaux ${ }^{10}$, I. Bertini ${ }^{32}$, S. Besse ${ }^{7}$, B. Carry ${ }^{11}$, G. Cremonese ${ }^{12,32}$, V. Da Deppo ${ }^{13,32}$, B. Davidsson ${ }^{5}$, S. Debei ${ }^{8,32}$, M. De Cecco ${ }^{14}$, J. De Leon ${ }^{6}$, F. Ferri ${ }^{32}$, S. Fornasier ${ }^{9,33}$, M. Fulle ${ }^{15}$, S. F. Hviid ${ }^{1}$, R. W. Gaskell ${ }^{16}$, O. Groussin ${ }^{2}$, P. Gutierrez ${ }^{6}$, W. $\mid l p^{17}$, L. Jorda ${ }^{2}$, M. Kaasalainen ${ }^{18}$, H. U. Keller ${ }^{19}$, J. Knollenberg ${ }^{20}$, R. Kramm ${ }^{1}$, E. Kührt ${ }^{20}$, M. Küppers ${ }^{11}$, L. Lara ${ }^{6}$, M. Lazzarin ${ }^{3}$, C. Leyrat ${ }^{9}$, J. J. Lopez Moreno $^{6}$, S. Magrin ${ }^{3}$, S. Marchi ${ }^{21,32}$, F. Marzari ${ }^{22,32}$, M. Massironi ${ }^{23,32}$, H. Michalik ${ }^{24}$, R. Moissl ${ }^{1,11}$, G. Naletto ${ }^{25,32}$, F. Preusker ${ }^{20}$, L. Sabau ${ }^{26}$, W. Sabolo ${ }^{6}$, F. Scholten ${ }^{20}$, C. Snodgrass ${ }^{1}$, N. Thomas ${ }^{27}$,

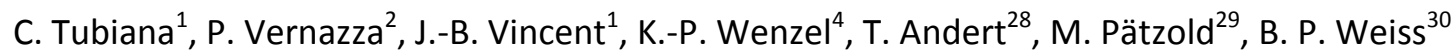

${ }^{1}$ Max-Planck-Institut für Sonnensystemforschung, Max-Planck-Strasse 2, 37191 Katlenburg-Lindau, Germany ${ }^{2}$ Laboratoire d'Astrophysique de Marseille, CNRS and Université de Provence, 38 Rue Frédéric Joliot-Curie, 13388 Marseille, France

${ }^{3}$ University of Padova, Department of Astronomy, Vicolo dell'Osservatorio 3, 35122 Padova, Italy

${ }^{4}$ Research and Scientific Support Department, European Space Agency, 2201 Noordwijk, The Netherlands

${ }^{5}$ Department of Physics and Astronomy, Uppsala University, 75120 Uppsala, Sweden

${ }^{6}$ Instituto de Astrofísica de Andalucía - CSIC, 18080 Granada, Spain

${ }^{7}$ Department of Astronomy, University of Maryland, Maryland 20742-2421, USA

${ }^{8}$ Department of Mechanical Engineering - University of Padova, Via Venezia 1, 35131 Padova, Italy

${ }^{9}$ LESIA, Observatoire de Paris, 5 Place Jules Janssen, 92195 Meudon, France

${ }^{10}$ LATMOS, CNRS/UVSQ/IPSL, 11 Boulevard d'Alembert, 78280 Guyancourt, France

${ }^{11}$ European Space Astronomy Centre, European Space Agency, Villanueva de la Cañada, Madrid, Spain

${ }^{12}$ INAF - Osservatorio Astronomico di Padova, Vicolo dell'Osservatorio 5, 35122 Padova, Italy

${ }^{13}$ CNR-IFN UOS Padova LUXOR, Via Trasea 7, 35131 Padova, Italy

${ }^{14}$ UNITN, Università di Trento, Via Mesiano, 77, 38100 Trento, Italy

${ }^{15}$ Osservatorio Astronomico de Trieste, 34014 Trieste, Italy

${ }^{16}$ Planetary Science Institute, 1700 East Fort Lowell, Suite 106, Tucson, AZ 85719, USA

${ }^{17}$ Institute for Space Science, National Central University, 32054 Chung-Li, Taiwan

${ }^{18}$ Department of Mathematics, Tampere University of Technology, 33101 Tampere, Finland

${ }^{19}$ Institute for Geophysics and Extraterrestrial Physics, TU Braunschweig, 38106 Braunschweig, Germany

${ }^{20}$ Institut für Planetenforschung, DLR, Rutherfordstrasse 2, 12489 Berlin, Germany

${ }^{21}$ Universite de Nice - Sophia Antipolis, Observatoire de la Cote d'Azur, CNRS, 06304 Nice, France

${ }^{22}$ Department of Physics - University of Padova, Via Marzolo 8, 35131 Padova, Italy

${ }^{23}$ Dipartimento di Geoscienze - Università di Padova, Via Gradenigo 6, 35131 Padova, Italy

${ }^{24}$ Institut für Datentechnik und Kommunikationsnetze, 38106 Braunschweig, Germany

${ }^{25}$ Department of Information Engineering - University of Padova, Via Gradenigo 6, 35131 Padova, Italy

${ }^{26}$ Instituto Nacional de Técnica Aeroespacial, 28850 Torrejon de Ardoz, Spain

${ }^{27}$ Physikalisches Institut der Universität Bern, Sidlerstrasse 5, 3012 Bern, Switzerland

${ }^{28}$ Institut für Raumfahrttechnik, Universität der Bundeswehr München, Neubiberg, Germany

${ }^{29}$ Rheinisches Institut für Umweltforschung, Abt. Planetenforschung, Universität zu Köln, Cologne, Germany

${ }^{30}$ Department of Earth, Atmospheric, and Planetary Sciences, MIT, Cambridge, MA, USA

${ }^{31}$ PAS Space Research Center, Bartycka 18A, 00-716 Warsaw, Poland

${ }^{32}$ CISAS - G. Colombo, Università di Padova, Via Venezia 15, 35131 Padova, Italy

${ }^{33}$ Universite Paris Diderot, Sorbonne Paris Cité, 4 Rue Elsa Morante, 75205 Paris, France

* To whom correspondence should be addressed. E-mail: sierks@mps.mpg.de 
Images obtained by the OSIRIS cameras onboard Rosetta reveal that 21 Lutetia has a complex geology and one of the highest asteroid densities measured so far, $(3.4 \pm 0.3) \mathrm{g} / \mathrm{cm}^{3}$. The North Pole region is covered by a thick layer of regolith, which is seen to flow in major landslides associated with albedo variation. Its geologically complex surface, ancient surface age and high density suggest that Lutetia is most likely a primordial planetesimal. This contrasts with smaller asteroids visited by previous spacecraft, which are probably shattered bodies, fragments of larger parents, or re-accumulated rubble piles.

The ESA Rosetta mission flew by asteroid Lutetia on July 10, 2010, with a closest approach distance of $3170 \mathrm{~km}$. Lutetia was chosen because of its size and puzzling surface spectrum (1,2). The OSIRIS imaging system on board Rosetta (3) took 462 images, in 21 broad- and narrow-band filters extending from 240 to $1000 \mathrm{~nm}$, through both its narrow-angle (NAC) and wide-angle (WAC) cameras. These images covered more than $50 \%$ of the asteroid surface, mostly of the northern hemisphere (Figs. S1, S2). The resolved observations started 9 hours 30 minutes before the closest approach (CA) and finished 18 minutes after CA. At CA, the asteroid filled the field of view of the NAC with a spatial scale of $\sim 60 \mathrm{mpx}^{-1}$. The observations reveal a morphologically diverse surface, indicating a long and complex history.

We modeled the global shape of Lutetia, combining two techniques: stereo-photoclinometry (4) using 60 NAC and WAC images, and inversion of a set of 50 photometric light curves and of contours of adaptive-optics images $(5,6)$. The asteroid's overall dimensions are $(121 \pm 1) \times(101 \pm 1) \times(75 \pm 13) \mathrm{km}^{3}$ along the principal axes of inertia. The North Pole direction is defined by a right ascension of $51.8^{\circ} \pm 0.4^{\circ}$ and a declination of $+10.8^{\circ} \pm 0.4^{\circ}$, resulting in an obliquity of $96^{\circ}$. From the global shape model, we derived a volume of $(5.0 \pm 0.4) \times 10^{5} \mathrm{~km}^{3}$. The volume error is well constrained by i) the dynamical requirement of principal-axis rotation, ii) the existence of ground-based adaptive optics images from viewing directions other than that of the flyby, and iii) the pre-flyby Knitted Occultation, Adaptive-optics and Light-curves Approach (KOALA) model (5), which matched the shape model of the imaged part within $5 \%$, giving us confidence that this model is accurate at this level for the southern hemisphere of Lutetia not seen during the flyby. The volume-equivalent-diameter of Lutetia is $(98 \pm 2) \mathrm{km}$. Combining our volume estimate with the mass of $(1.700 \pm 0.017) \times 10^{18} \mathrm{~kg}$ measured by the Radio Science Investigation (7), we obtained a density of $(3.4 \pm 0.3) \mathrm{g} / \mathrm{cm}^{3}$. This value is higher than that found for most nonmetallic asteroids, whose bulk densities are in the range 1.2 to $2.7 \mathrm{~g} / \mathrm{cm}^{3}$, well below the average 
grain density of their likely meteoritic analogs. Such low densities imply large macroporosities (8) that are associated with "rubble pile" asteroids (9).

Using crater density, cross-cutting and overlapping relationships and the presence of deformational features like faults, fractures and grooves, we have identified five major regions on the surface observed during the closest approach. Two regions (Pannonia and Raetia) imaged at lower resolution were defined on the basis of sharp morphological boundaries as crater walls and ridges (Fig. 1, supporting online material, SOM, for details). The surface is covered in regolith, with slopes below the angle of repose for talus almost everywhere, but large features reveal the underlying structure. A cluster of craters close to the pole in the Baetica region is one of the most prominent features of the Northern hemisphere. The most heavily cratered, and therefore oldest, regions (Noricum and Achaia) are separated by the Narbonensis region, which is defined by a crater of $\sim 55 \mathrm{~km}$ in diameter (Fig. 2). This crater (Massilia) contains several smaller units, and is deformed by grooves and pit-chains, indicating modifications that took place after its initial formation. Another large impact crater is seen close to the limb (Raetia region). A sub-parallel ridge formation is seen close to the terminator. A number of scarps and linear features (grooves, fractures, and faults) transecting several small craters (Figs. 2, S3), are organized along systems characterized by specific orientations for each region and with no obvious relationships with the major craters. However, in the Noricum region a prominent scarp bounds a local topographic high where lineaments run almost parallel to the scarp itself and to the rims of the crater cluster in Baetica. High-resolution topography models produced by stereo image processing (10) show that one long (> $10 \mathrm{~km}$ ) groove in the Noricum region (Fig. 2C, S4) is roughly $100 \mathrm{~m}$ deep and on a local topographic high. The linear features are similar in appearance to those on the Martian moon Phobos, commonly interpreted as resulting from a large impact (11). On 433 Eros, the existence of similar grooves has been interpreted as evidence of competent rock below the regolith, although this asteroid is thought to be heavily fractured $(12,13,14)$. Recent work suggests that cracks can be supported in very low strength material on a body as small as Eros (15). The pattern of grooves on Lutetia suggests strain structures or fractures within a body of considerable strength.

Lutetia is heavily cratered, although the crater spatial density varies considerably across the imaged hemisphere. We have identified more than 350 craters with diameter between $600 \mathrm{~m}$ and $55 \mathrm{~km}$ which allowed us to the determine Lutetia's crater retention age by measuring the crater size-frequency distribution (SFD). We chose to perform the crater count on the Achaia region because it is a remarkably flat area imaged with uniform illumination conditions. In this region, we counted 153 craters over an 
area of $2800 \mathrm{~km}^{2}$. We compared Achaia's SFD with those for asteroids 253 Mathilde and 243 Ida (Fig. 3). At large crater sizes $(>10 \mathrm{~km})$, the crater SFD of Achaia is quite similar to that of Ida, while Mathilde is only slightly less cratered. There are $\sim 2$ or $\sim 3$ times less craters at $1 \mathrm{~km}$ diameter than on Ida or Mathilde, respectively. At very small sizes $(<1 \mathrm{~km})$, there is a strong depletion of craters. Asteroids as large as Lutetia can be globally affected by seismic shaking; this argument has been used to explain the depletion of $<200$ m diameter craters on $\operatorname{Eros}(13,16)$, but cannot explain the observed paucity of craters with diameters up to $5-8 \mathrm{~km}$ (17). The apparent break in the SFD at this size range is statistically significant: according to the Kolmogorov-Smirnov test, the probability that the observed crater SFD (for D $>0.8 \mathrm{~km}$ ) is consistent with a simple hard rock scaling law model (i.e. an approximately linear crater SFD, Fig. 3C) is only 3\%.

Small crater obliteration by Massilia crater ejecta seems unlikely given that the Achaia region does not show a systematic decrease in crater density with increasing distance to Massilia. A possible explanation for the break is a transition in the physical properties of the target. Small craters, which only affect the upper layers, form in shattered material. Larger craters, able to excavate to greater depth, form in competent rock. We therefore modeled a gradual transition in the crater scaling law as strength and density increase with depth in a fractured layer (18). We determined the depth of this layer by fitting the model to the observed crater SFD $(19,20)$ (Fig. 3C). For typical rock properties [supporting online material (SOM) text] the depth of the fractured layer is $\sim 3 \mathrm{~km}$. Based on this model, and using the lunar chronology as calibration (20), we find a crater retention age of (3.6 \pm 0.1$)$ Ga for Achaia.

Scaling laws (21) and hydrocode simulations performed with iSALE (22) show that the impactor that produced Massilia had a diameter $\sim 8 \mathrm{~km}$. According to the simulation, this impact heavily fractured but did not completely shatter Lutetia. The current main belt impact rate suggests that such an impact occurs every $\sim 9 \mathrm{Ga}$; therefore the impact may have occurred relatively early in the Solar System history when the collisional environment in the asteroid belt was more intense. The early occurrence of such an impact is in agreement with the crater retention age for Lutetia.

The Baetica region is partially covered by smooth material that is interpreted as ejecta from the $21 \mathrm{~km}$ diameter crater cluster. The images show evidence that older, smaller craters were partially buried by the ejecta. The depth of the ejecta blanket is estimated to be up to 600 $\mathrm{m}$ based on the depth-todiameter ratios of these buried craters. The asymmetric shape of the $21 \mathrm{~km}$ crater cluster may be the result of internal inhomogeneity. Indeed preexisting planes of weakness in bedrocks may control the final crater shape and facilitate detachment of blocks and their emplacement within ejecta deposits 
(23). The crater interior (Fig. 2B) shows a great variety of deposits: smooth and fine deposits with boulders, gravitational taluses, and landslide accumulations. Ejecta blocks have been recorded on other asteroids (13), and Phobos (24). On Lutetia, approximately 200 blocks of up to $300 \mathrm{~m}$ in dimension were found around the central crater region alone. Their steep size distribution (a power law equation with exponent of -5 ) is comparable to that seen on Eros (13). The presence of boulders adjacent to another impact site in the Pannonia region suggests that boulder generation is a common feature of large impacts on Lutetia, and points to excavation of shattered bedrock. The landslides appear to have been emplaced after the boulders and may have been triggered by further impacts.

To investigate the reflectance properties of the surface, OSIRIS obtained images (including several color sequences) at different asteroid rotational phases, and over a range of phase angles from $0.15^{\circ}$ to $156^{\circ}$. The slope of the phase curve (Fig. S5) for phase angles between $5^{\circ}$ and $30^{\circ}$ is $0.030 \mathrm{mag} /{ }^{\circ}$ for the $631 \mathrm{~nm}$ filter. The Lutetia disk-integrated geometric albedo was measured to be $(0.194 \pm 0.006)$ at $631 \mathrm{~nm}$ and $(0.169 \pm 0.009)$ at $375 \mathrm{~nm}$, giving an average value in the $V$ band $(550 \mathrm{~nm})$ of $(0.19 \pm 0.01)$ and a Bond albedo of (0.073 \pm 0.002$)$.

We computed disk-resolved reflectivity maps at $10^{\circ}$ solar phase angle using the 3D shape model and light-scattering theory (25) in order to remove the effect of variation in illumination conditions due to the topography (Fig. 4). We detected variations of the surface reflectivity at $647 \mathrm{~nm}$ wavelength. The most important variegations are located inside the crater cluster in the Baetica region (Fig. 4A), where reflectivity varies up to $30 \%$ between the darkest and brightest areas. Small spatial variations in reflectivity are also present on surrounding terrain (Fig. 4B) but with a much lower contrast. In Baetica, a clear correlation is found with the local surface slope. Landslide flows or possible rock outcrops appear much brighter than the accumulation areas or surrounding cratered terrains. This suggests either a different texture of regolith or that space weathering modified the surface of oldest areas, while young surfaces have been less exposed to solar radiation. Similar variations of reflectivity have been already observed on Eros, where a strong correlation between the spectral slope and the down slope movement of regolith was found (13). Disk-integrated spectrophotometry obtained $1 \mathrm{~h}$ before CA reveals a flat and featureless spectrum, with a moderate spectral slope in the visible range ( $3 \% / 10^{3} \AA$ between 536 and $804 \mathrm{~nm}$ ), in agreement with spectra obtained from VIRTIS-Rosetta spectrometer (26) and ground-based spectra taken at similar phase angle (Fig. S6). These data are consistent with both particular types of carbonaceous chondrite meteorites, namely $\mathrm{CO} 3$ and CV3 $(1,27)$, and enstatite chondrites (ECs) (28). Average bulk densities $(8,34)$ range from 2.96 to $3.03 \mathrm{~g} / \mathrm{cm}^{3}$ for $\mathrm{CO}$ and $\mathrm{CV}$ meteorites and $3.55 \mathrm{~g} / \mathrm{cm}^{3}$ 
for ECs. If Lutetia were composed purely of EC material, this would imply a bulk asteroid macroporosity of $\sim 0-13 \%$ (given the uncertainty range on Lutetia's density). The low densities of CO and CVs preclude the possibility of a pure composition of either meteorite group. If Lutetia's surface were made of these materials, this would suggest that the interior may be differentiated (29).

These macroporosities for Lutetia clearly exclude a rubble pile structure, which typically have macroporosities $>25-30 \%$ (9). Such a high porosity structure is also inconsistent with the extensive ejecta blankets observed around the large craters (30). If Lutetia is undifferentiated, these porosities would also exclude a completely shattered but coherent structure (total porosity in the range 15 to $25 \%$ ) (31). Partial differentiation (29) could permit much higher grain densities in the interior and therefore higher porosity and a heavily fractured body. It is therefore likely that Lutetia has survived the age of the Solar System with its primordial structure intact; i.e. it has not been disrupted by impacts. This interpretation is consistent with the current view that the collisional lifetime against catastrophic destruction of bodies with diameters $\geq 100 \mathrm{~km}$ exceeds the age of the Solar System (32). The network of curvilinear features, the crater morphology and the crater SFD discussed above both indicate that Lutetia's interior has considerable strength and relatively low porosity compared to that expected for primordial aggregates of fine dust. One possibility is that Lutetia is partially differentiated, with a fractured but unmelted chondritic surface overlaying a higher density sintered or melted interior (29). In any case, Lutetia is closer to a small planetesimal than to the smaller asteroids seen by previous missions, which are thought to be shattered or rubble pile minor bodies. 


\section{Figures}

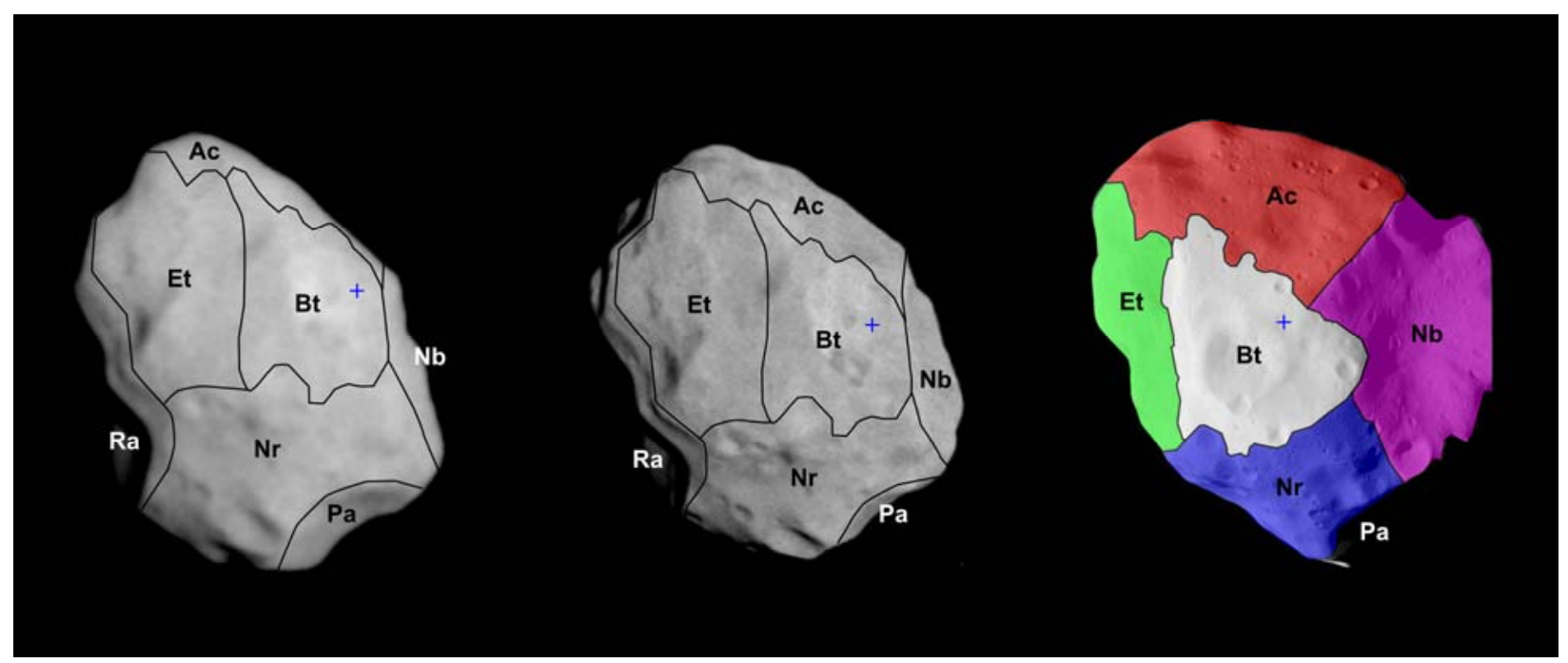

Fig. 1. Regions on Lutetia. Three images taken at $-60,-30$, and -3 minutes prior to closest approach showing the different regions: Bt. Baetica, Ac. Achaia, Et. Etruria, Nb. Narbonensis, Nr. Noricum, Pa. Pannonia, Ra. Raetia. The images were taken at distances of 53, 27, and $3.5 \times 10^{6} \mathrm{~m}$ and phase angles of $8^{\circ}, 4^{\circ}$, and $52^{\circ}$. The resolutions of each image are approximately 1000, 500, and $60 \mathrm{~m} \mathrm{px}^{-1}$; Lutetia has been scaled to appear approximately the same size in each panel. The North Pole is indicated by the blue cross. 


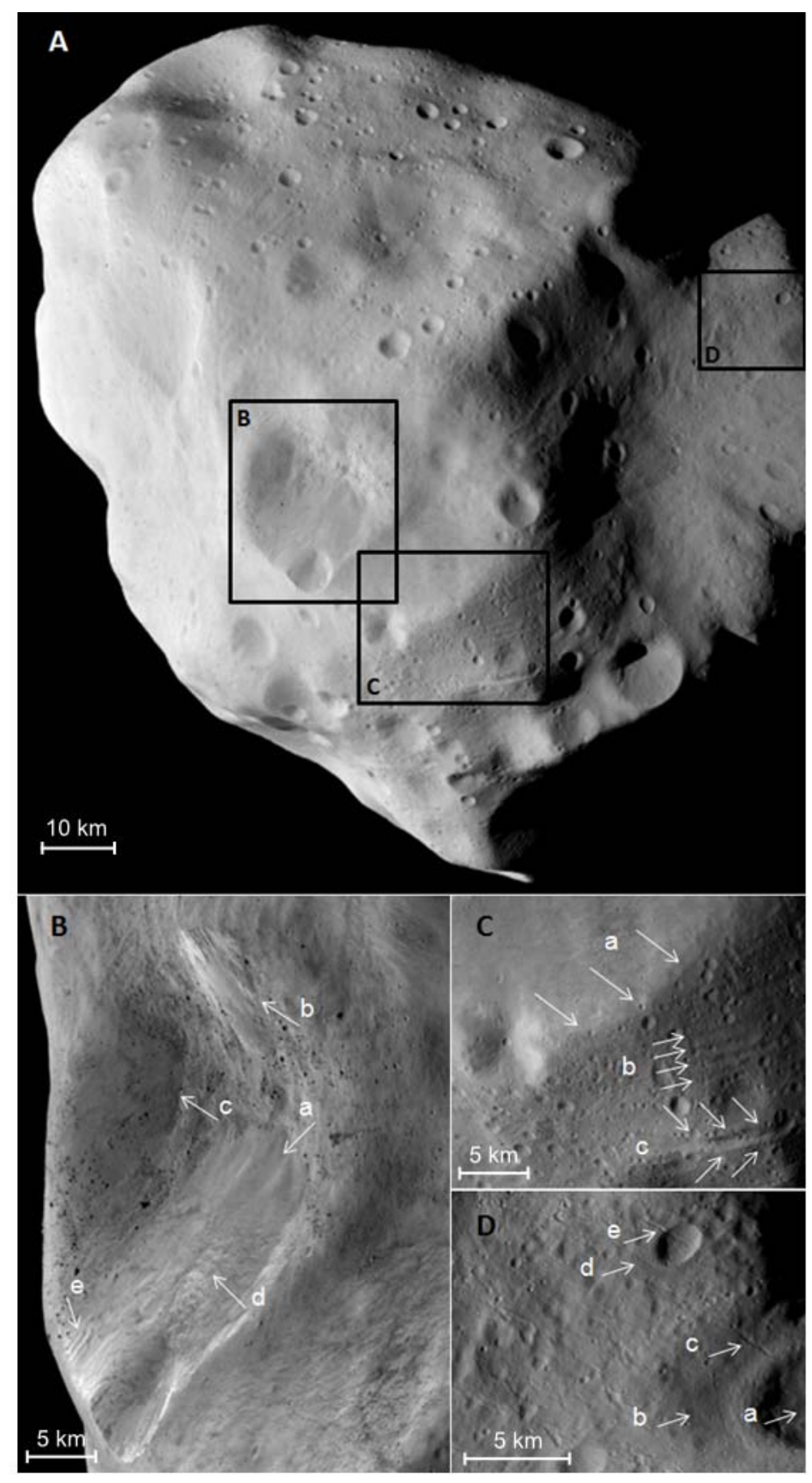

Fig. 2. Surface features. (A) Closest approach image, with insets showing details under different illumination conditions. (B) The central $21 \mathrm{~km}$ diameter crater cluster in Baetica. Arrows $a, b$, and c point to landslides. Landslides $\mathrm{a}$ and $\mathrm{b}$ appear to have buried the boulders that are pervasive within the crater (average density of 0.4 boulders $\mathrm{km}^{-2}$ ). Landslide $b$ may have exposed a rocky outcrop. A similar possible outcrop is seen opposite (e). Note the mottled appearance of the material at point $d$. (C) The boundary 
between Baetica (young terrain associated with the central crater cluster) and Noricum (old terrain) is extremely well-defined in some places as indicated by the arrows $a$. Arrows $b$ and $c$ highlight curvilinear features. (D) Arrows c, d, and e point to further curvilinear features on the surface of Lutetia. In the Narbonensis region, most curvilinear features show this orientation. Note how the curvilinear features cut the crater and its rim. Feature c cuts through the debris apron (b) of the crater (a). This implies that these linear features are younger than the craters, or impact into an area with existing large scale cracks and subsequent regolith movement. 

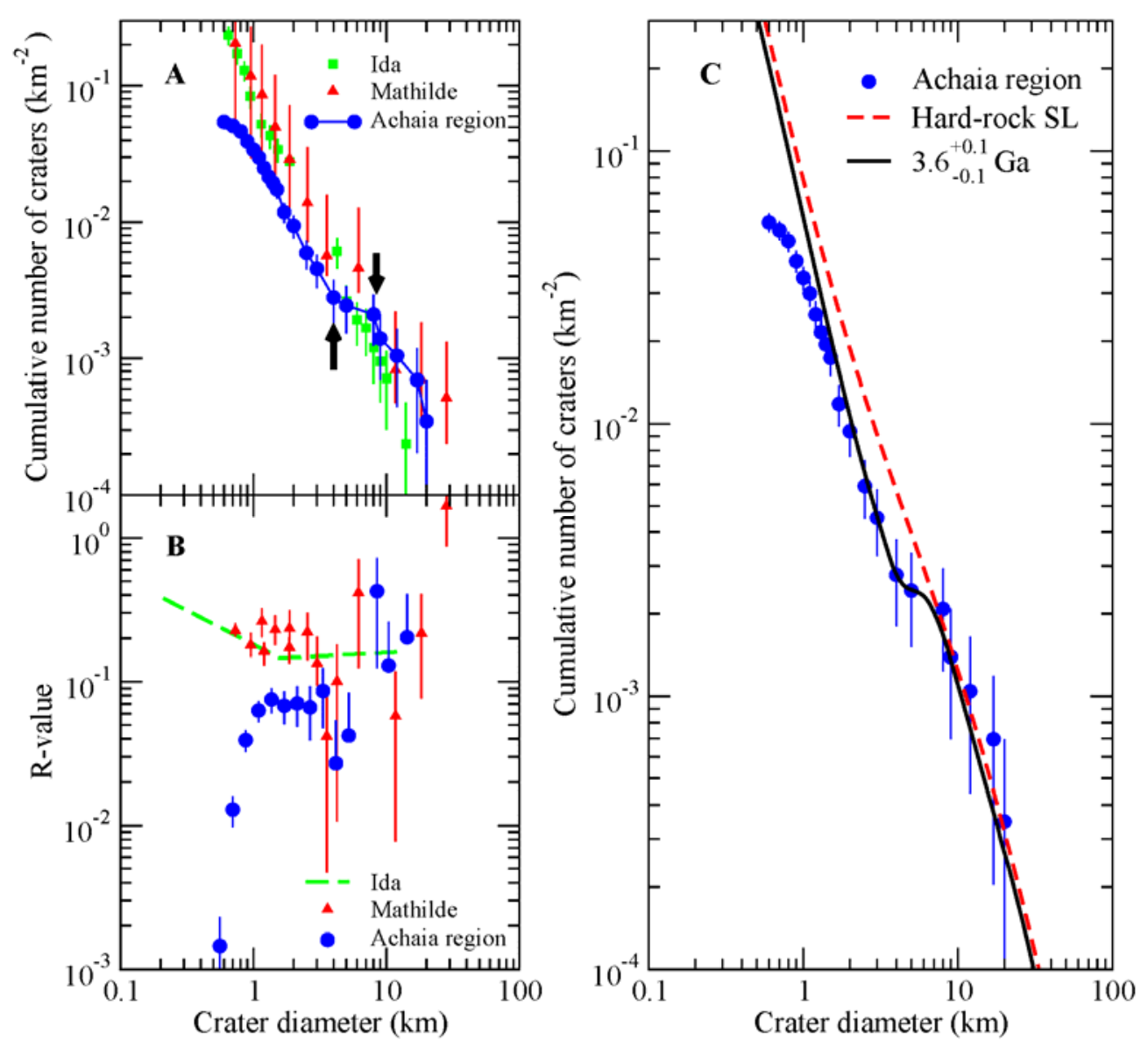

Fig. 3. Crater SFD. (A) Cumulative crater SFD of Achaia region compared with those for Ida and Mathilde, the second and third largest asteroids imaged by spacecraft so far, respectively [data from (17)]. The arrows indicate the suggested break at 5-8 km in the Achaia crater SFD. (B) SFD shown in A expressed in terms of relative (R) values (cumulative crater SFD normalized to a power law with exponent -2). $\mathrm{R}$ values for Ida are not published, but the overall trend (dashed line) was computed from the published cumulative distribution. (C) Achaia crater SFD model fit. The dashed red curve represents a fit of the largest craters of the distribution ( $D>10 \mathrm{~km}$ ) obtained using current models for the main belt asteroid size distribution (33) and the crater scaling law for hard-rock (21). The black curve is the best fit achieved by a two layer (fractured material over competent rock) model, which gives a crater retention age of (3.6 \pm 0.1$) \mathrm{Ga}$. 


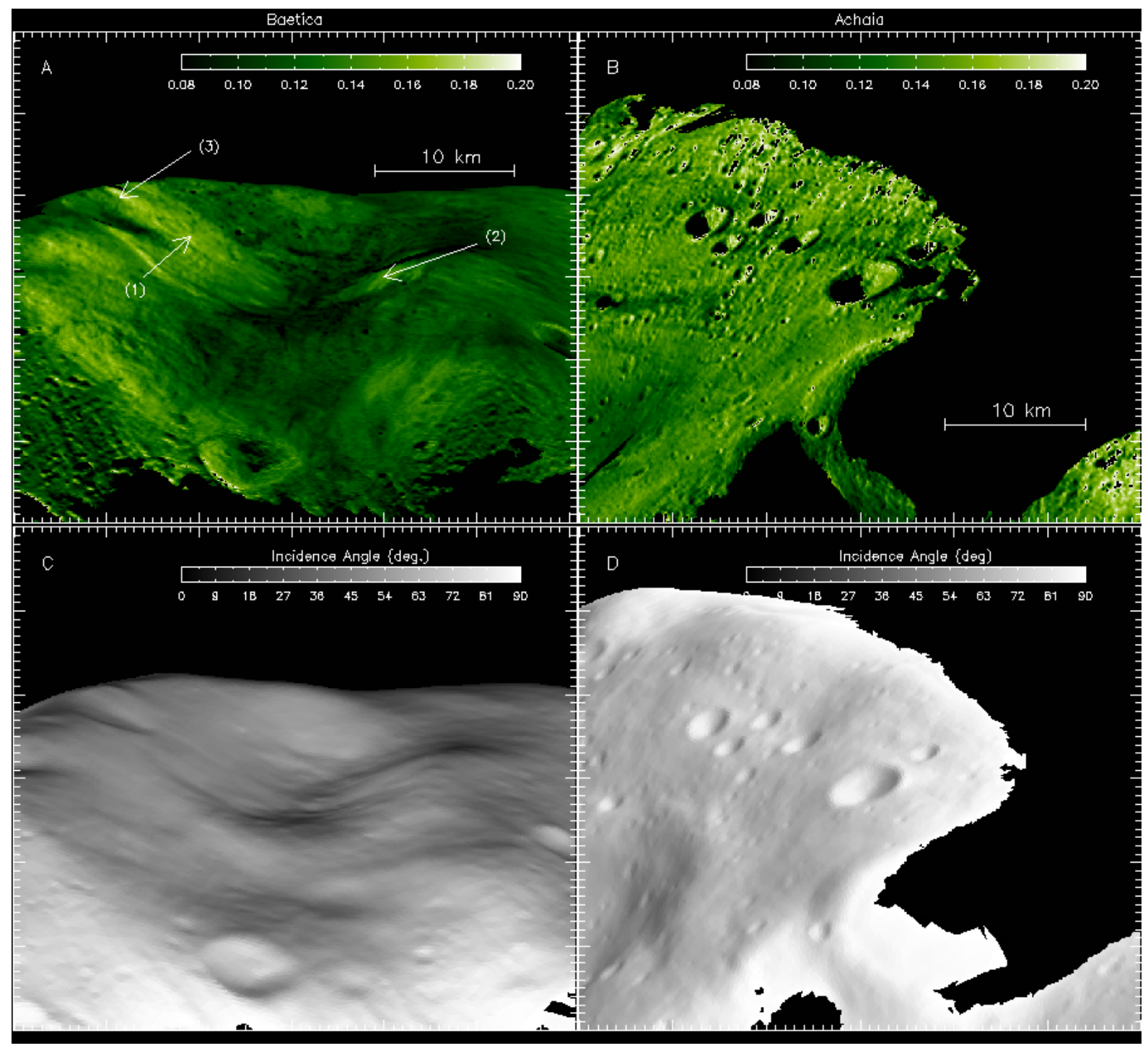

Fig. 4. Slope corrected reflectivity maps (A+B) and incidence angles maps (C+D). Images at $647 \mathrm{~nm}$ of parts of the Baetica $(\mathbf{A}+\mathbf{C})$ and Achaia $(\mathbf{B}+\mathbf{D})$ regions that have been photometrically corrected using Hapke bi-directional reflectance theory (25) to remove the effect of different angles of incidence and emission for different local slopes, leaving variations in brightness due only to local albedo variations (resolution $60 \mathrm{~m} \mathrm{px}^{-1}$ ). During the photometric correction, the Hapke model parameters describing the single scattering albedo, the coherent back scattering, the shadow hiding, the surface roughness and the asymmetric factor were all fixed to the value that best reproduced the overall surface reflectivity. The images are corrected to a solar phase angle of $10^{\circ}$ for both Baetica and Achaia (the original phase angles for these regions were $\sim 70^{\circ}$ to $95^{\circ}$ ). This phase angle was arbitrarily chosen to avoid the opposition 
effect that may affect the reflectivity near $0^{\circ}$ phase angle. Large variations are visible in the younger Baetica region, while the older Achaia region is more uniform (aside from a dark streak associated with a crater in the left of the image). The landslide indicated by 1 and possible outcrops 2 and 3 in Baetica have a reflectivity up to $30 \%$ brighter than the accumulation area.

\section{References and Notes}

[1] M. A. Barucci, M. Fulchignoni, in Rosetta: ESA's Mission to the Origin of the Solar System, R. Schulz, C. Alexander, H. Boehnhardt, K.-H. Glassmeier (Eds.), (Springer) 55-68 (2009).

[2] M. Lazzarin, S. Magrin, S. Marchi, E. Dotto, D. Perna et al., Mon. Not. R. Astron. Soc. 408, 1433-1437 (2010).

[3] H. U. Keller, C. Barbieri, P. Lamy, H. Rickman, R. Rodrigo et al., Space Sci. Rev. 128, 433-506 (2007).

[4] R. W. Gaskell, O. S. Barnouin-Jha, D. J. Scheeres, A. S. Konopliv, T. Mukai, et al., Meteoritics \& Planetary Science 43, 1049-1061 (2008).

[5] B. Carry, M. Kaasalainen, C. Leyrat, W. J. Merline, J. D. Drummond, et al., Astron. Astrophys. 523, id.A94 (2010).

[6] M. Kaasalainen, Inverse Problem and Imaging. 5, 37-57 (2011).

[7] M. Pätzold et al. Science, XXX, XXXX (2011) 
[8] G. Consolmagno, D. Britt, R. Macke, Chemie der Erde Geochemistry 68, 1-29 (2008).

[9] We use "rubble pile" to mean a "strengthless aggregate held together by gravity only", following the definition of S. L. Wilkison, M. S. Robinson, P. C. Thomas, J. Veverka, T. J. McCoy, S. L. Murchie, L. M.

Prockter, D. K. Yeomans, Icarus 155, 94-103 (2002)

[10] J. Oberst, F. Preusker, R. J. Phillips, T. R. Watters, J. W. Head, M. T. Zuber, S.C. Solomon, Icarus 209, 230-239 (2010).

[11] P. Thomas, J. Veverka, A. Bloom, T. Duxbury, J. Geophys. Res. 84, 8457-8477 (1979).

[12] J. Veverka, M. Robinson, P. Thomas, S. Murchie, J. F. Bell, et al., Science 289, 2088-2097 (2000)

[13] R. J. Sullivan, P. C. Thomas, S. L. Murchie, M. S. Robinson, in Asteroid III by W.F. Bottke Jr., A. Cellino, P. Paolicchi, and R. P. Binzel (Eds.), (University of Arizona Press, Tucson) 331-350 (2002).

[14] A. F. Cheng, in Asteroid III by W. F. Bottke Jr., A. Cellino, P. Paolicchi, and R. P. Binzel (Eds.), University of Arizona Press, Tucson, 351-366 (2002).

[15] E. Asphaug, Annu.Rev. Earth Planet. Sci. 37, 413 (2009)

[16] J. E. Richardson, H.J. Melosh, R. J. Greenberg, D. P. O'Brien, Icarus 179, 325-349 (2005)

[17] D. P. O'Brien, R. Greenberg, J. E. Richardson, Icarus 183, 79-92 (2006).

[18] S. Marchi, M. Massironi, E. Martellato, L. Giacomini, L. M. Prockter, Planet. Space Sci., arXiv:1105.5272, DOI 10.1016/j.pss.2011.06.007 (2011).

[19] S. Marchi, C. Barbieri, M. Küppers, F. Marzari, B. Davidsson, et al., Planet. Space Sci. 58, 1116-1123 (2010).

[20] S. Marchi, S. Mottola, G. Cremonese, M. Massironi, E. Martellato, Astron. J. 137, 4936-4948 (2009).

[21] K. A. Holsapple, K. R. Housen, Icarus 187, 345-356 (2007).

[22] K. Wünnemann, G. S. Collins, H. J. Melosh, Icarus 180, 514 (2006).

[23] H.J. Melosh, in Impact cratering- a geological process (Oxford University Press) 84 (1989).

[24] P. C. Thomas, J. Veverka, R. Sullivan, D. P. Simonelli, M. C. Malin, et al., J. Geophys. Res. 105, 15091-15106 (2000). 
[25] B. Hapke, Icarus 157, 523-534 (2002).

[26] A. Coradini et al. Science, XXX, XXXX (2011).

[27] M. A. Barucci, S. Fornasier, E. Dotto, P. Lamy, L. Jorda, et al., Astron. Astrophys. 477, 665-670 (2008).

[28] P. Vernazza, R. Brunetto, R. P. Binzel, C. Perron, D. Fulvio, et al., Icarus 202, 477-486 (2009).

[29] L. T. Elkins-Tanton, B. P. Weiss, and M. T. Zuber (2011) Chondrites as samples of differentiated planetesimals, Earth Planet. Sci. Lett., 305, 1-10.

[30] K. R. Housen and K. A. Holsapple, Icarus 163, 102-119 (2003).

[31] D. T. Britt, D. Yeomans, K. Housen, G. Consolmagno, in Asteroid III by W. F. Bottke Jr., A. Cellino, P. Paolicchi, and R. P. Binzel (Eds.), University of Arizona Press, Tucson, 485-500 (2002).

[32] A. Morbidelli, W. F. Bottke, D. Nesvorný, H. F. Levison, Icarus 204, 558-573 (2009).

[33] W. F. Bottke, D. D. Durda, D. Nesvorný, R. Jedicke, A. Morbidelli, et al., Icarus 175, 111-140 (2005).

[34] R. J. Macke, G. J. Consolmagno, D. T. Britt, M. L. Hutson, Meteorit. Planet. Sci. 45, 1513-1526 (2010).

\section{Acknowledgements}

OSIRIS was built by a consortium of the Max-Planck-Institut für Sonnensystemforschung, KatlenburgLindau, Germany, CISAS - University of Padova, Italy, the Laboratoire d'Astrophysique de Marseille, France, the Instituto de Astrofísica de Andalucia, CSIC, Granada, Spain, the Research and Scientific Support Department of the European Space Agency, Noordwijk, The Netherlands, the Instituto Nacional de Técnica Aeroespacial, Madrid, Spain, the Universidad Politéchnica de Madrid, Spain, the Department of Physics and Astronomy of Uppsala University, Sweden, and the Institut für Datentechnik und Kommunikationsnetze der Technischen Universität Braunschweig, Germany.

The support of the national funding agencies of Germany (DLR), France (CNES), Italy (ASI), Spain (MEC), Sweden (SNSB), and the ESA Technical Directorate is gratefully acknowledged.

We thank the Rosetta Science Operations Centre and the Rosetta Mission Operations Centre for the successful flyby of (21) Lutetia. 


\section{Supporting Online Material (SOM) - Sierks et al.}

\section{"Images of Asteroid 21 Lutetia: A Remnant Planetesimal from the Early Solar System"}

Here we present extra figures showing the area of Lutetia's surface seen by the OSIRIS cameras, the location of the curvilinear features and the average photometric properties of the surface. We also describe the parameters of the model used to recreate the shape of the crater SFD.

The images from OSIRIS cover almost the entire northern hemisphere. The images are projected onto two maps (Figs. S1, S2) that show the resolved area in cylindrical and polar stereographic projection. The first shows the entire area covered, while the second shows the details better with less distortion because the highest resolution images were of the North Pole region. The western hemisphere (regions Raetia and Etruria) was seen to lower latitudes at low resolution during the approach, while the eastern hemisphere (Narbonensis) was seen at higher resolution but at high phase angle. This is why there are more shadows seen in the projected map in the East - these maps have not been corrected for shadowing and local slopes. The southern region of Lutetia was not seen as it is in shadow due to the high obliquity $\left(96^{\circ}\right)$. This area was in permanent (winter) darkness at the time of the fly-by. The shape model in this region is completed based on the ground based KOALA model (5), and the main uncertainty on the shape and volume is due to the fact that the length of the c-axis (pole-to-pole distance) is not measured directly by OSIRIS.

Figures S3 and S4 highlight details of curvilinear features. The first is a map of the features overlaid on the closest approach image showing the global layout and orientation of features, and their large sizes. The second is an image generated from the 3D model of the surface that shows a perspective view along one of the troughs.

The disk averaged photometry is used to measure the overall reflectance properties of the asteroid. Figure $\mathrm{S} 5$ shows the phase curve. The parameters in the International Astronomical Union (IAU) H-G photometric system are $H=7.243$ and $G=0.132$. Figure $S 6$ shows the OSIRIS photometry (points) compared with a previous spectrum. We confirm the featureless nature of the spectrum in the visible and NIR wavelength range.

The geological subdivision of the regions on Lutetia is detailed on pg. 8. Figure S7 provides insight in the definition of regions for the Baetica and Achaia region as well as for the separation of Etruria from Noricum. 


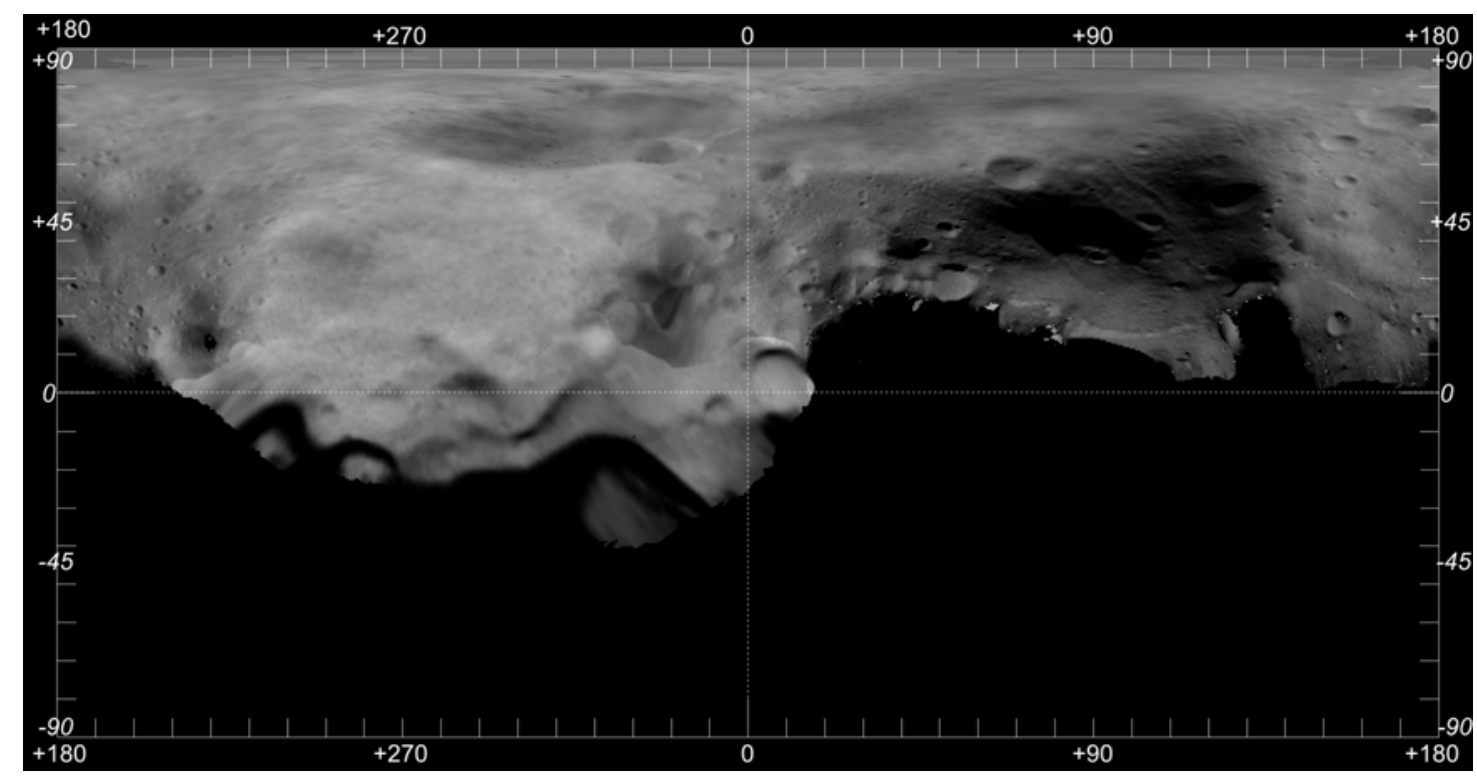

Fig. S1. Cylindrical projection of Lutetia. Composite map of several images acquired in the last hour before the closest approach, in a cylindrical projection. This represents the total area viewed by the spacecraft during the flyby (more than $50 \%$ of the asteroid surface). To compensate for changes in resolution and Sun/Rosetta geometry, colors have been manually adjusted for the display and do not represent any real photometric measurement. For example, the dark area in Narbonesis region (around $+45,+270$ ) is due to shadowing in this large depression in high phase angle images, not due to darker material. 


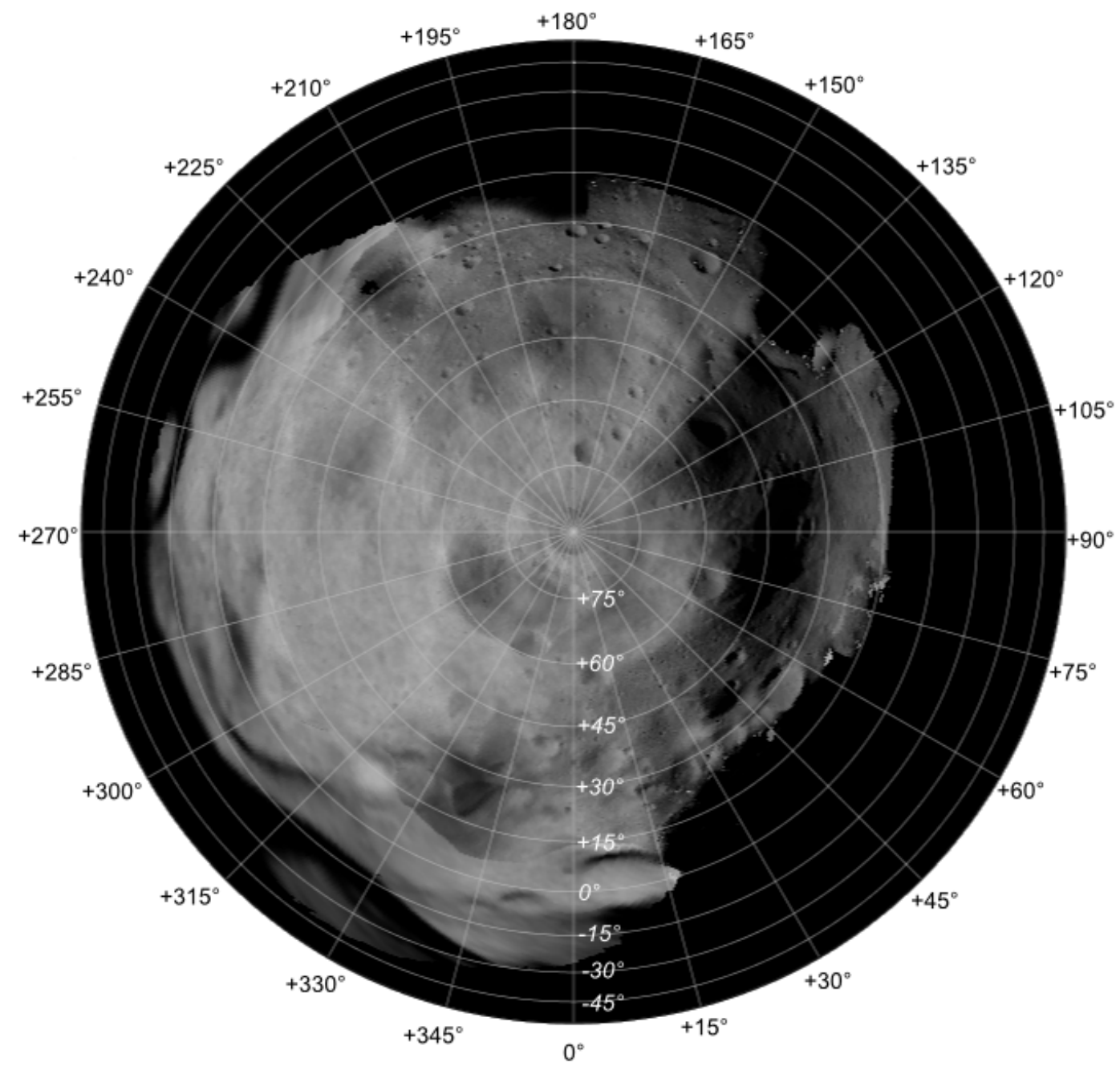

Fig. S2. Polar stereographic projection. Same map as in Fig. S1, in an azimuthal equal-area projection centered on the North Pole, and covering latitudes from $+90^{\circ}$ to $-40^{\circ}$. 


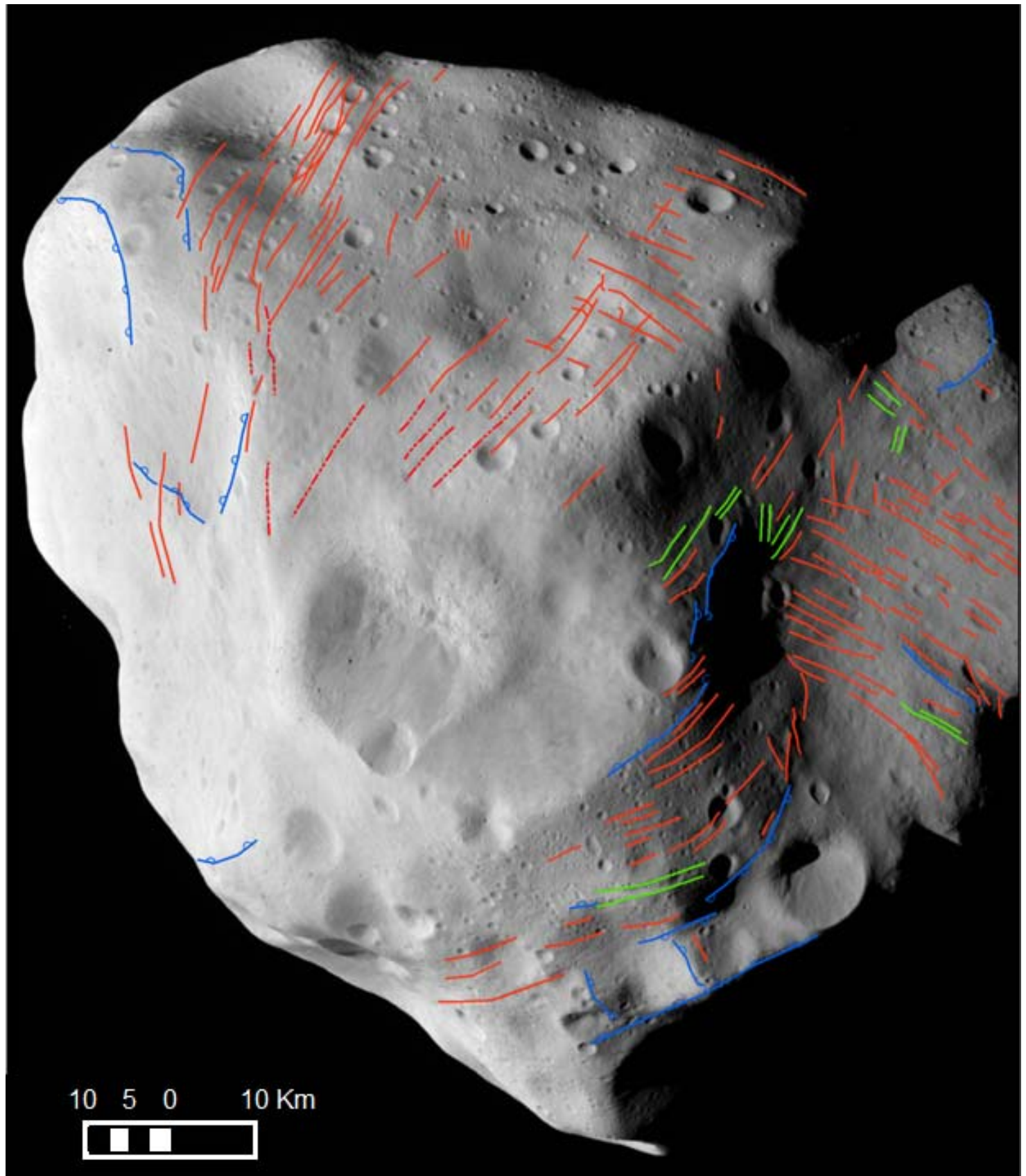

Fig. S3. Curvilinear features on the surface of Lutetia. The feature orientations are strongly locationdependent. Different colors correspond to different feature types: blue lines mark scarps; green lines indicate troughs; red solid lines trace generic curvilinear features (fractures, faults, grooves, pitchains) and red dotted lines indicate curvilinear features buried by Baetica region ejecta. 


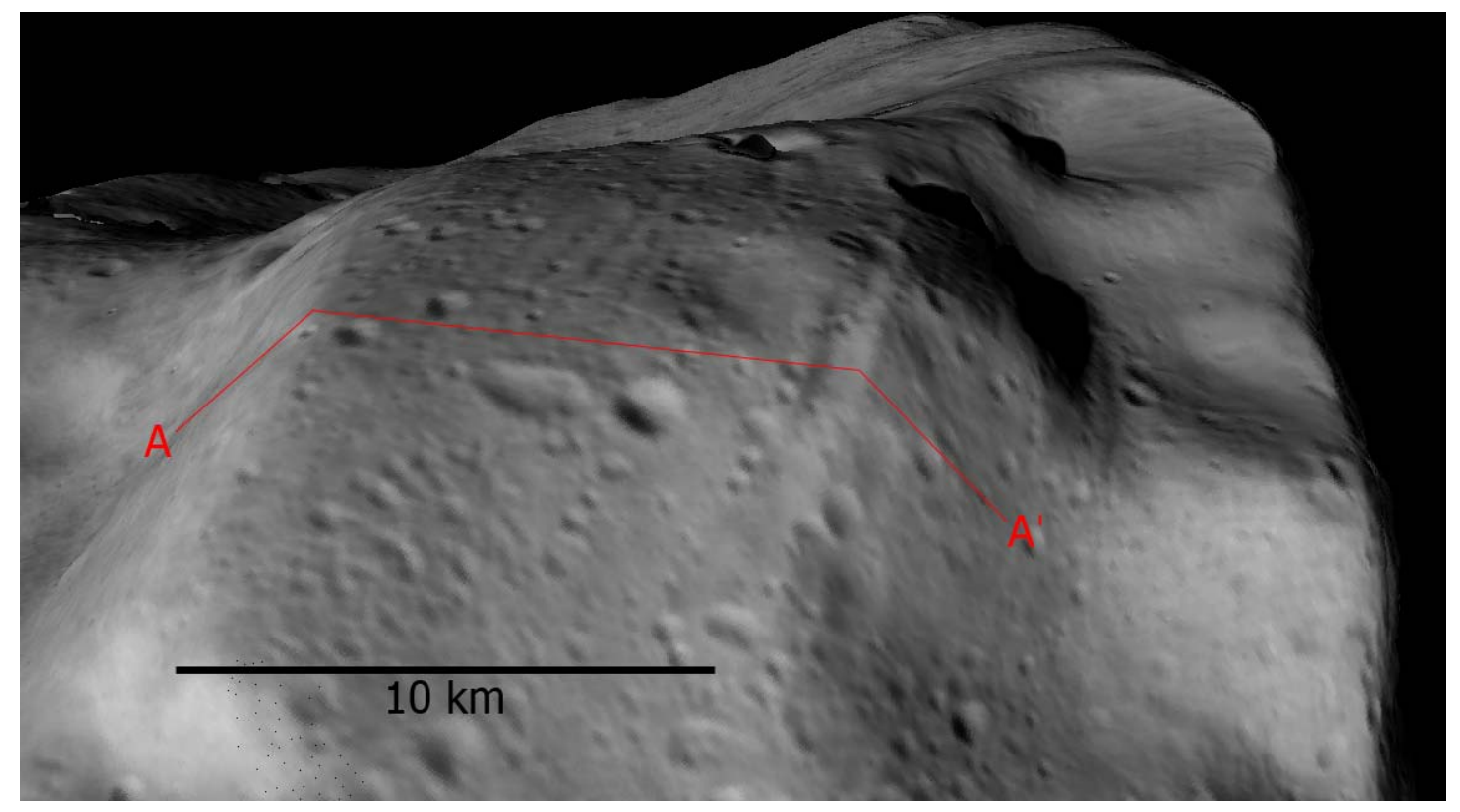

Fig. S4. Perspective view. This image is generated from a digital terrain model (DTM) looking along the trough c in Fig. 2C. On the left, is the edge of the North Pole crater cluster. Note also the grooves in the older Noricum region are parallel to the trough. The trough is at least $10 \mathrm{~km}$ long and roughly $100 \mathrm{~m}$ deep. It is on a local topographic high and the appearance suggests that a geological uplift has led to a surface chasm. The change in slope (as measured in the DTM, relative to a sphere) seen along the profile $A-A^{\prime}$ is quite extreme, being $45^{\circ}$ to the left and $23^{\circ}$ to the right. It is possible that this was a second-order effect of an impact in the Baetica region. If so, this would also point to a low porosity, consolidated, interior. The perspective view implies that the scale bar is appropriate only for the foreground. The DTM was derived by stereo-photogrammetric processing from 10 NAC images near closest approach. These images cover almost the entire northern hemisphere of Lutetia. 
21 Lutetia, OI-WAC

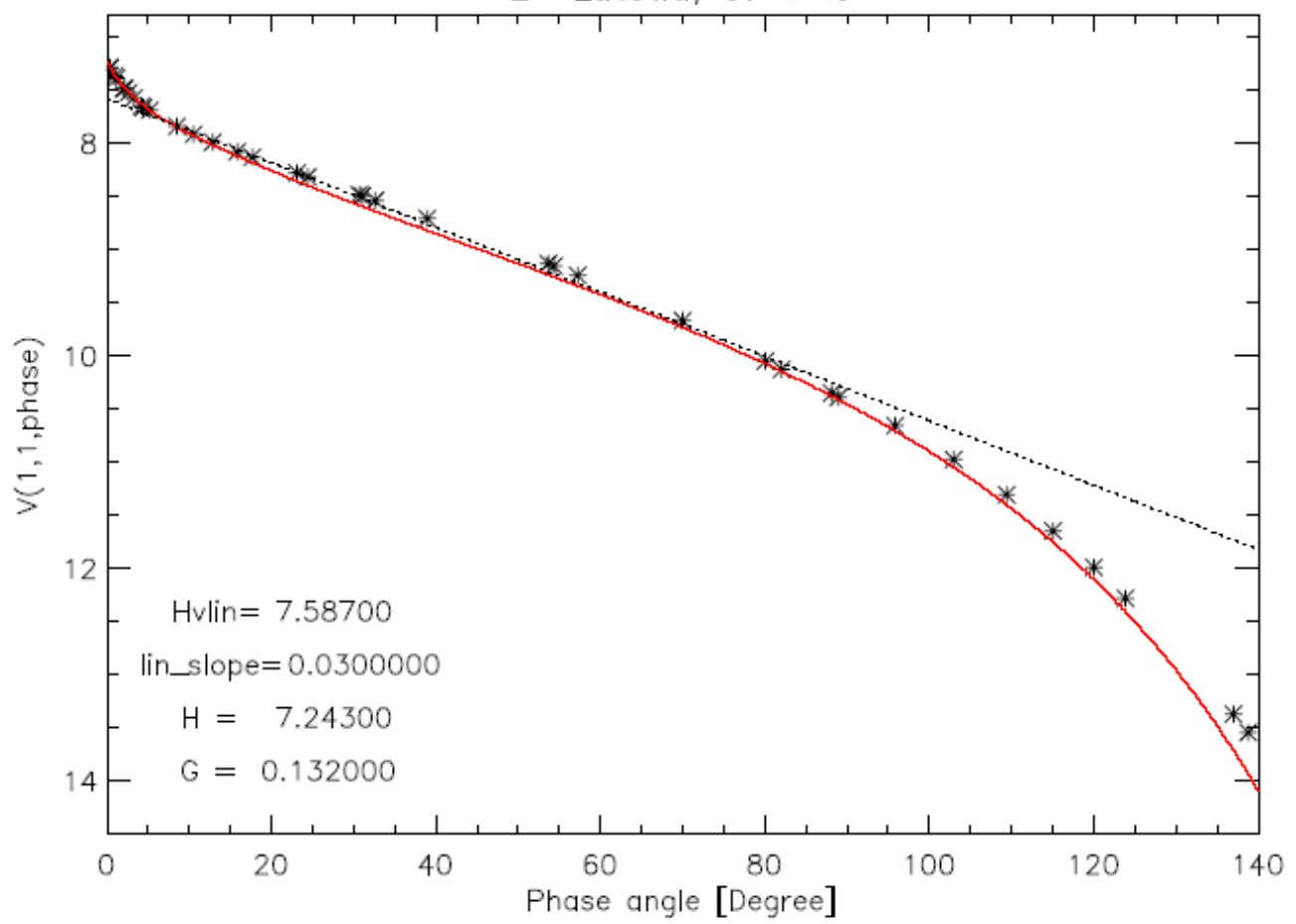

Fig. S5. Phase curve. The disk integrated brightness of Lutetia measured at different phase angles, using the OI $(631 \mathrm{~nm})$ filter. The dotted line shows a best fit to the linear fit, which has a slope of $0.03 \mathrm{mag} /{ }^{\circ}$, and the red line shows the best fit in the IAU H-G system. The fit parameters are given in the figure. Note the measurement of the opposition surge made possible by the spacecraft trajectory, with a minimum phase angle of $0.15^{\circ}$. 


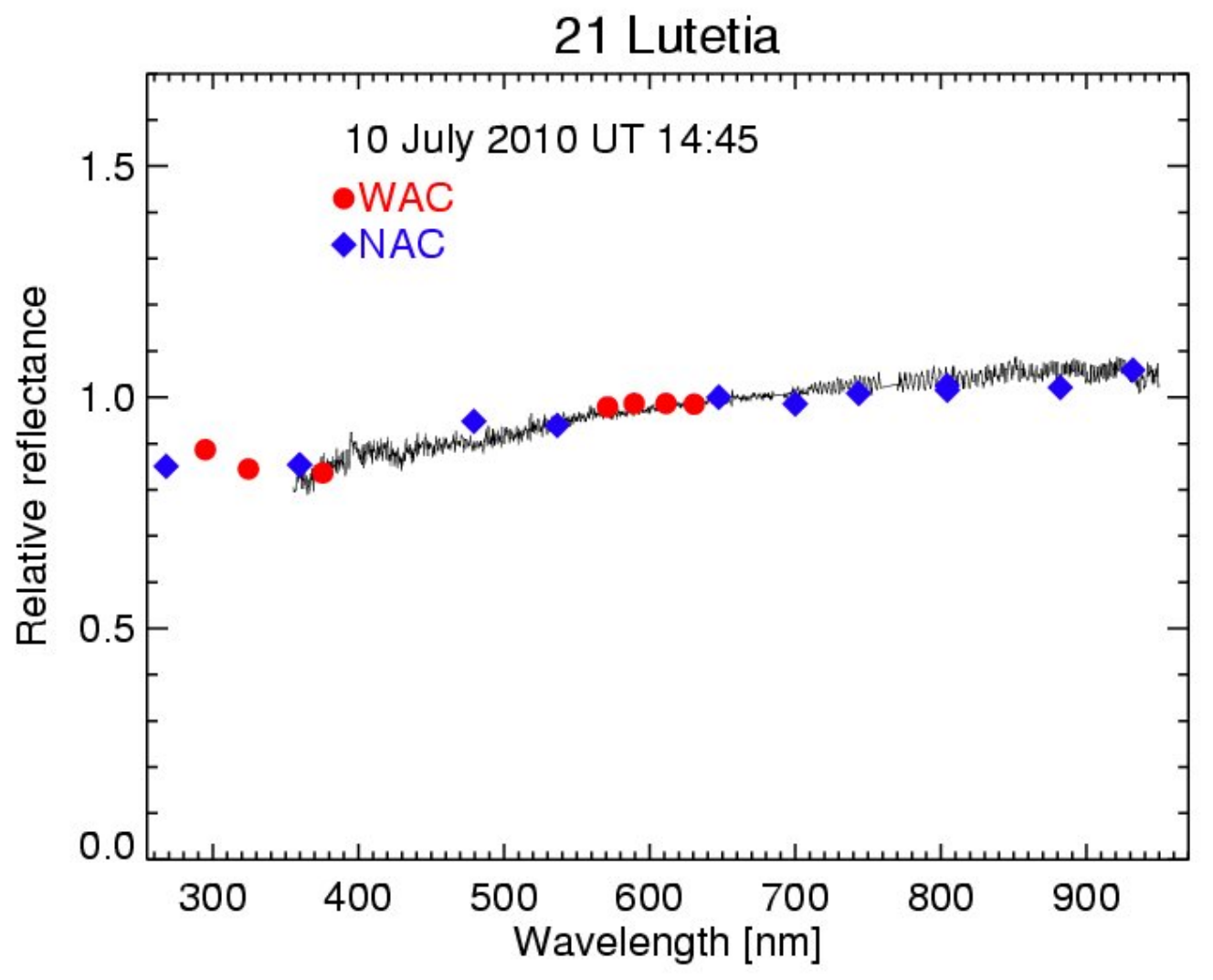

Fig. S6. OSIRIS spectrophotometry. Photometric points from OSIRIS (red stars from WAC camera, blue diamonds from NAC) obtained from disk integrated images $1 \mathrm{~h}$ before CA, at UT 14:45. The Rosetta-Lutetia distance was $55000 \mathrm{~km}$ and the phase angle $7.74^{\circ}$ (NAC $100 \mathrm{px}$ diameter, WAC $\sim 20 \mathrm{px}$ diameter). For comparison, a ground based spectrum is shown. It was taken with the $3.6 \mathrm{~m}$ Telescopio Nazionale Galileo on 15/16 Nov 2004 at very similar phase angle ( $8^{\circ}$ ) (36). 


\section{Lutetia regions}

The long geological history of Lutetia is recorded on its highly varied surface which displays several geological units. The geological subdivision has been made by means of the crater density, crosscutting and overlapping relationship and the presence of deformational features like faults, fractures and grooves. On the imaged surface, we identified seven major geological units, called regions that may be subdivided into minor sub-units. The regions display clear morphological border and/or cross-cutting and embayment relationships.

The Achaia and Noricum regions are the more heavily cratered and show several linear features most of which are grooves, fractures and troughs. The Etruria region has different orientation in space with respect to the plateau-like area of Achaia and the strongly deformed Noricum region; it is separated from these latter by morphological highs like the large rims of Berna and Roma craters. Despite the bad illumination condition, in Etruria it is still possible to note a highly cratered surface, a prominent scarp and several possible fractures. All these units are partially covered by the smooth material of the Baetica geological system which can be related to recent impacts at the Northern pole (North Pole crater cluster). This major unit lacks of linear feature and is characterized by an extremely low crater density.
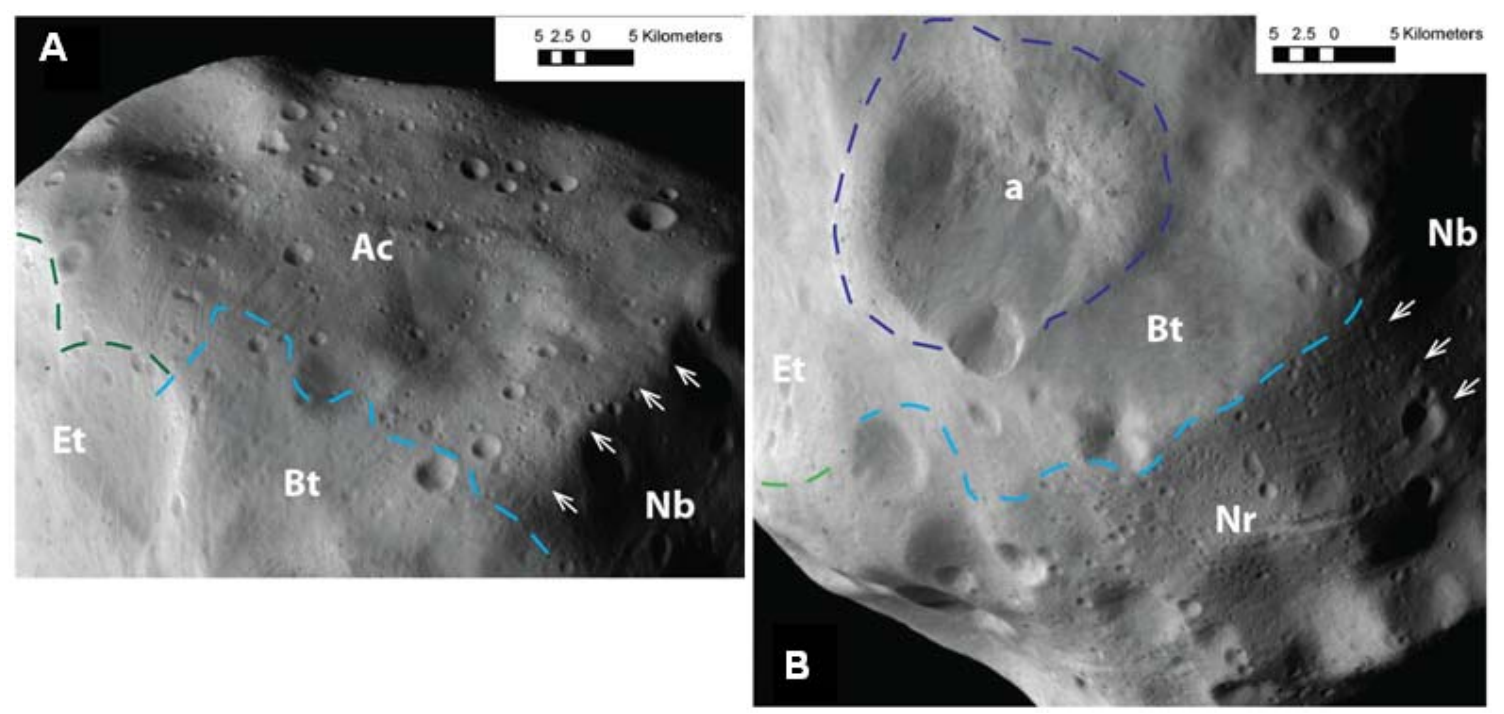

Fig. S7. Definition of regions. A: Achaia region (Ac) and its geological boundaries. The green dashed line indicates the asymmetric ridge separating Etruria (Et) from Achaia, the white arrows show the crater rim of Massilia (55 km in diameter) cutting the Achaia region and separating it from the Narbonensis $(\mathrm{Nb})$ region, the light blue dashed line shows the limit of the ejecta blankets of the Baetica region crater cluster covering the Achaia plateau (note how the linear features of Achaia are partly or totally buried by these deposits). B: Noricum ( $\mathrm{Nr}$ ) and Baetica (Bt) regions boundaries. The craters and ejecta of the Baetica region, characterized by a low crater density, cut and overlap the older Noricum region (higher crater density); a light blue dashed line highlights this boundary. Within the Baetica region, crater "a" ( $21 \mathrm{~km}$ of diameter) and related ejecta overlap the other major craters of the region. White arrows indicate the locations where Massilia crater wall cuts the Noricum region, whereas the green dashed line shows the ridge separating Etruria from Noricum region. 


\section{Crater SFD Model}

In the model used to describe the apparent break in the crater SFD, we assumed a linear increase of both density and material strength in the top layer of fractured material. For the rest of the interior we assumed constant values. The values for the surface are: material strength: $3.010^{4} \mathrm{dyne} / \mathrm{cm}^{2}$ and density: $2 \mathrm{~g} / \mathrm{cm}^{3}$, while at the bottom of the layer (which for the best fit case is of a depth of $\sim 3 \mathrm{~km}$ ) they are: material strength: $210^{8}$ dyne $/ \mathrm{cm}^{2}$ and density: $3.4 \mathrm{~g} / \mathrm{cm}^{2}$. The assumptions of the model (uniform increase of the parameters for the top layer) come from previous studies $(18,19)$. The material strength and density of the surface are from lunar regolith, while the interior density value comes for our measurements, and the interior material strength value is the one usually used in asteroid studies (35).

\section{Additional References}

[35] E. Asphaug, Moore, J. M., Morrison, D., Benz, W., Nolan, M. C., Sullivan, R. J., Mechanical and Geological Effects of Impact Cratering on Ida, Icarus, 120, 158 (1996).

[36] I. N. Belskaya, S. Fornasier, Yu. N. Krugly, V. G. Shevchenko, Astron. Astrophys. 515, id.A29 (2010). 